\title{
The Diffusion, Impacts, Adoption and Usage of ICTs upon Society Mini-track Introduction
}

\author{
Jyoti Choudrie \\ University of Hertfordshire \\ Hertfordshire Business School \\ Hatfield, Hertfordshire \\ AL10 9EU \\ e-mail: j.choudrie@herts.ac.uk
}

\author{
Sherah Kurnia \\ David Sundaram \\ School of Computing and Information Department of Information Systems and \\ Systems \\ University of Melbourne \\ Australia \\ e-mail: sherahk@unimelb.edu.au
}

Since the Covid-19 pandemic has affected society, economies and organizations significantly, the adoption, use and diffusion of Information and Communication Technologies (ICTs) in various aspects of human life has become extremely important to manage. The aim of this minitrack is to offer a global perspective of how ICTs are being used, adopted, used and diffused within society including households, enterprises of any size, government and social communities. ICTs are wide ranging from the infrastructure of Broadband (high speed internet) to mobile devices, online social networks, sharing platforms, Artificial Intelligence, Big Data Analytics, blockchain and other platforms enabled by the internet.

Adoption, usage and diffusion studies are prevalent in Information Systems (IS) research and offer an insight into many issues surrounding 'how', 'when' and 'what' technologies are being introduced and their impacts. By undertaking this stream of research, academics, industry and government agencies will learn of how ICTs are being utilized by various groups and communities in society and what measures are being undertaken to have households and the various social communities adopt and use the ICTs with a further consideration of the impacts of the ICTs. Case studies, experiments, longitudinal and cross- sectional, snapshot studies, literature reviews, empirical, comparative, and applied studies related to ICT use, adoption, impacts and diffusion are emerging on a daily basis.

This mini track facilitates discussion among global researchers about their latest research on technology adoption, acceptance, use, diffusion and impacts. The unit of analysis may include individuals, organizations, government agencies and the broader societies. The audience is expected to gain insights into the latest applications and use of ICTs, adoption challenges experienced by individuals, organizations, and governments, possible strategies to address current challenges, and approaches to manage the consequences of ICT adoption and diffusion within the societies.

This mini track has constantly received high-quality research papers related to ICT adoption, use, diffusion and impact. This year, we are delighted to present six innovative research papers that offer valuable insights and novel contribution to research and practice.

The first paper, Assessing the Impact of COVID-19 on Website Technology Penetration on Businesses in Rural Towns, investigated web technology (WT) adoption in five rural towns, 849 businesses prior to and during the COVID-19 pandemic. Longitudinal data was analyzed by town, technology, and economic activity. Findings 
revealed that COVID-19 resulted in significant growth in the adoption of online advertising, cloud computing, and ecommerce in rural businesses. Rural businesses located in commuter towns adopted more WTs than those in other rural towns. Highly digitalized sectors increased their WT adoption more than those reliant on physical products or human interaction. Similarly, those who already had high levels of WTs in place adopted more sophisticated technologies.

The second paper, Non-Adoption of Dynamic Pricing in Traditional Taxi Dispatch Organizations is a qualitative case study of Finnish dispatch organizations to investigate the reasons for the non-adoption of Dynamic pricing (DP) in rid-sharing organizations. Utilizing IT artifacts as an analytical lens, the study identifies ten aspects related to the technology, the structure and the context within which DP is embedded that help explain why it has not been adopted. The authors propose that DP in the taxi industry should be seen more broadly than just as "Uber-type" real-time $\mathrm{DP}$, as it has been viewed in previous literature.

The third paper, Coping with IT! Antecedents and Consequences of Technostress in E-Commerce investigates which technology-induced stressors create technostress in an online store and how they affect purchase intention. Findings showed a negative indirect effect of technostress on consumers' purchasing intention, mediated by consumers' perception of website quality, website trust, and choice of coping strategy. The paper contributes to technostress, coping and e-commerce literature and extends research by presenting empirically validated technology-induced stressors together with insights into the mechanism of a transactional technostress-model in the context of e-commerce.
The fourth paper, Diffusion and Adoption of Technology amongst Small and Medium Enterprises during COVID-19- with a focus on Internet of Things presents a conceptual study of small and medium sized enterprises (SMEs) technology adoption and diffusion with a focus on impact on IoT adoption during COVID-19. The paper proposes that the pandemic has acted as a legitimizing force for technology adoption and diffusion amongst SMEs.

The fifth paper, An Innovation Resistance Perspective on Seller Resistance to Digital Device Recycling Platforms (DDRP) develops a deeper understanding of the reasons underlying seller resistance to the DDRP that is grounded in the innovation resistance theory (IRT). Although DDRPs offer new opportunities for commerce and sustainability, challenges exist to usher in their wide adoption. The research model of this study was validated using data collected from 307 potential sellers of used digital devices. The model helps understand why some sellers resist DDRPs.

The final paper, Understanding and exploring employees' use of an enterprise social network within a large retail organization explores and explains the use of an Enterprise Social Network within a large retail organization. A qualitative approach of interpretivism and analytical technique of thematic analysis and open coding drawn from grounded theory were employed. The context of this study was a large retail organization in the UK. The study identifies interrelationship between resistance, workaround, organizational culture, trust and working practices and their overall impact on the ESN usage behavior. It also helps employees and employers to use ESN as a cost-effective way of identifying the knowledge of other employees, appreciating that knowledge, contacting employees easily, and pursuing information. 